\title{
Heteronuclear decoupling interference during symmetry-based homonuclear recoupling in solid-state NMR
}

\author{
Ildefonso Marin-Montesinos a, Darren H. Brouwer ${ }^{\mathrm{a}}$, Giancarlo Antonioli ${ }^{\mathrm{a}}$, \\ Wai Cheu Lai ${ }^{\mathrm{a}}$, Andreas Brinkmann ${ }^{\mathrm{b}}$, Malcolm H. Levitt ${ }^{\mathrm{a}, *}$ \\ ${ }^{a}$ School of Chemistry, University of Southampton, Southampton SO17 1BJ, UK \\ ${ }^{\mathrm{b}}$ Institute for Molecules and Materials, Radboud University Nijmegen, 6525 ED Nijmegen, The Netherlands
}

Received 2 June 2005; revised 13 July 2005

Available online 19 September 2005

\begin{abstract}
We examine the influence of continuous-wave heteronuclear decoupling on symmetry-based double-quantum homonuclear dipolar recoupling, using experimental measurements, numerical simulations, and average Hamiltonian theory. There are two distinct regimes in which the heteronuclear interference effects are minimized. The first regime utilizes a moderate homonuclear recoupling field and a strong heteronuclear decoupling field; the second regime utilizes a strong homonuclear recoupling field and a weak or absent heteronuclear decoupling field. The second regime is experimentally accessible at moderate or high magic-angle-spinning frequencies and is particularly relevant for many realistic applications of solid-state NMR recoupling experiments to organic or biological materials.
\end{abstract}

(C) 2005 Elsevier Inc. All rights reserved.

Keywords: Symmetry-based recoupling; Decoupling; Double-quantum NMR; Magic-angle spinning; Average Hamiltonian theory

\section{Introduction}

Recoupling methods reintroduce specific nuclear spin interactions that are normally averaged out by magicangle-spinning (MAS), so that both the high spectral resolution afforded by MAS and the structural information provided by these nuclear spin interactions are preserved. The symmetry theory for rotor-synchronized pulse sequences [1] allows the design of pulse sequences that selectively recouple desired classes of nuclear spin interaction under MAS conditions. Pulse sequences based on the two classes of symmetries, $C N_{n}^{v}$ and $R N_{n}^{v}$, have been employed for a wide variety of experiments [1-42]. In particular, some of the most important and successful applications of symmetry-based sequences have involved double-quantum (DQ) homonuclear

\footnotetext{
${ }^{*}$ Corresponding author.

E-mail address: mhl@soton.ac.uk (M.H. Levitt).
}

dipolar recoupling. These applications include two-dimensional DQ correlation spectroscopy $[4,9,10,14,22$, $23,33]$, high-order multiple-quantum excitation in solids $[13,15,36,41,42]$, and the estimation of internuclear distances $[26,39]$, torsional angles $[3,5,31]$, and motional order parameters [38].

For organic and biological samples, the most common case concerns recoupling of ${ }^{13} \mathrm{C}$ nuclei introduced by isotopic labelling (similar conditions apply to the recoupling of ${ }^{15} \mathrm{~N}$ or ${ }^{31} \mathrm{P}$ nuclei). In organic solids, the homonuclear interactions between ${ }^{13} \mathrm{C}$ nuclei are usually accompanied by strong heteronuclear dipolar interactions with the surrounding ${ }^{1} \mathrm{H}$ nuclei. These heteronuclear interactions generally interfere with the homonuclear ${ }^{13} \mathrm{C}-{ }^{13} \mathrm{C}$ recoupling. The heteronuclear interference is normally suppressed by applying a strong resonant $\mathrm{RF}$ field on the ${ }^{1} \mathrm{H}$ channel while the symmetry-based recoupling sequence is applied to the ${ }^{13} \mathrm{C}$ channel. Most early work in this area was conducted at low spinning 
frequencies (around $6 \mathrm{kHz}$ or less), where good heteronuclear decoupling during homonuclear recoupling is achieved by maintaining a ratio of 2.5 or more between the ${ }^{1} \mathrm{H}$ and ${ }^{13} \mathrm{C}$ nutation frequencies [8-10]. This approach is generally not feasible at moderate to high spinning frequencies (around $10 \mathrm{kHz}$ or more), since the required $\mathrm{RF}$ field on the ${ }^{1} \mathrm{H}$ channel would be unacceptably high.

One approach to reduce heteronuclear interference at higher MAS frequencies is to use sequences with symmetries that require lower ratios of ${ }^{13} \mathrm{C}$ nutation frequency to MAS frequency. For example, the SPC5 [16] and the supercycled $\mathrm{C} 14_{4}^{5}$ sequences [18] require ${ }^{13} \mathrm{C}$ nutation frequencies that are 5 and 3.5 times the MAS frequency, respectively. Kristiansen et al. [35] showed that the symmetry class may be chosen to allow efficient ${ }^{13} \mathrm{C}$ dipolar recoupling at $30 \mathrm{kHz}$ spinning frequency in the presence of high power ${ }^{1} \mathrm{H}$ decoupling. The disadvantage of this approach is that the required symmetries have relatively small scaling factors for the homonuclear dipolar interactions and are often less robust with respect to the effects of RF inhomogeneity, isotropic chemical shift differences, and anisotropic chemical shift interactions.

Ishii [43] showed that some pulse sequences, such as fpRFDR (finite-pulse radio-frequency driven recoupling) function well at very high spinning frequencies (around $30 \mathrm{kHz}$ ) in the complete absence of a ${ }^{1} \mathrm{H}$ decoupling field. To some extent, this is expected, since heteronuclear dipolar interactions transform in the same way as the ${ }^{13} \mathrm{C}$ chemical shift anisotropy (CSA) under rotations of the ${ }^{13} \mathrm{C}$ spins by the RF field and by the sample rotation, providing that a ${ }^{1} \mathrm{H}$ RF field is absent. Since the ${ }^{13} \mathrm{C}$ CSA is compensated by most homonuclear recoupling sequences, it is not surprising that the heteronuclear interactions are suppressed without further intervention, under suitable conditions. However, in the case of fpRFDR, this "no-decoupling regime" is only reached for very fast sample rotation frequencies in the vicinity of $30 \mathrm{kHz}$. Many solid-state NMR applications require relatively large sample volumes, for which the maximum available spinning frequencies are currently limited to around $15 \mathrm{kHz}$.

Hughes et al. [44] demonstrated that the ${ }^{13} \mathrm{C}$ DQ filtering efficiency for $\left[{ }^{13} \mathrm{C}_{2}\right]$ glycine obtained with the POST-C7 ${ }_{2}^{1}$ sequence [7] without ${ }^{1} \mathrm{H}$ decoupling at $12 \mathrm{kHz}$ spinning frequency was comparable to that obtained with high power ${ }^{1} \mathrm{H}$ decoupling at $7 \mathrm{kHz}$ spinning frequency. This suggests that the no-decoupling regime appears at conveniently low spinning frequencies for the symmetry-based pulse sequences. This makes sense, since the symmetry-based design of these sequences ensures their high compensation for CSA, and by extension, for heteronuclear interactions in the absence of heteronuclear decoupling.

Fig. 1 shows that this effect also occurs for sequences belonging to the $R N_{n}^{v}$ symmetry class. The spectra shown
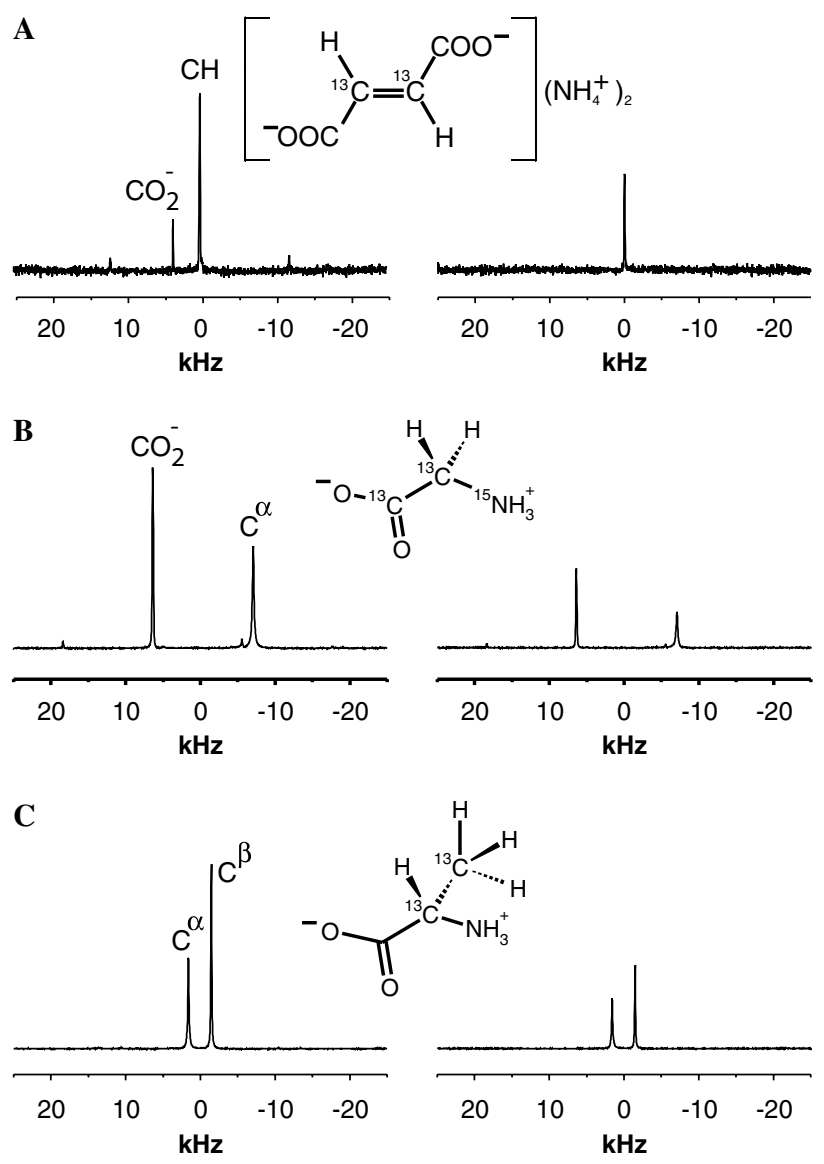

Fig. 1. Comparison of ${ }^{13} \mathrm{C}$ CP MAS NMR spectra (left column) with double-quantum filtered spectra (right column) for (A) 10\%-labelled diammonium- $\left[2,3-{ }^{13} \mathrm{C}_{2}\right]$ fumarate, (B) $\left[{ }^{15} \mathrm{~N},{ }^{13} \mathrm{C}_{2}\right]$ glycine, and (C) $\left[2,3-{ }^{13} \mathrm{C}_{2}\right]$ alanine. The double-quantum-filtered spectra were obtained at a spinning frequency of $\omega_{r} / 2 \pi=12 \mathrm{kHz}$ using the $R 20_{2}^{9}$ sequence without ${ }^{1} \mathrm{H}$ decoupling during the recoupling period.

in Fig. 1 demonstrate ${ }^{13} \mathrm{C}$ DQ filtering efficiencies greater than $35 \%$ in three ${ }^{13} \mathrm{C}_{2}$-labelled organic compounds at a MAS frequency of $12 \mathrm{kHz}$, using a pulse sequence with symmetry $R 20_{2}^{9}$, without using a heteronuclear decoupling field during the homonuclear recoupling sequence.

In this paper, we present an experimental and theoretical investigation of heteronuclear interference during ${ }^{13} \mathrm{C}$ homonuclear dipolar recoupling, for the case of double-quantum homonuclear recoupling pulse sequences of the symmetry class $R N_{n}^{v}$. We explore the effects of the spinning frequency, the nutation frequencies on both the ${ }^{13} \mathrm{C}$ and ${ }^{1} \mathrm{H}$ channels, and the symmetry numbers. For simplicity, we concentrate on $R N_{n}^{v}$ sequences applied in the presence of unmodulated (continuous-wave, CW) ${ }^{1} \mathrm{H}$ irradiation, applied on-resonance. We show that the interference caused by heteronuclear interactions may be simulated fairly accurately by numerical simulations and that the general features of heteronuclear decoupling during homonuclear recoupling sequences may be predicted by first-order average Hamiltonian theory. Heteronuclear interference 
with the $C N_{n}^{v}$ class of pulse sequences is considered briefly in the Supporting information.

\section{Pulse sequences}

\subsection{Symmetry-based recoupling}

This article concerns DQ ${ }^{13} \mathrm{C}$ homonuclear dipolar recoupling with symmetry-based $R N_{n}^{v}$ sequences, as sketched in Fig. 2. A pulse sequence with the symmetry $R N_{n}^{v}$ may be constructed as follows:

$R N_{n}^{v}=\left[R_{\phi} R_{-\phi}^{\prime}\right]^{N / 2}$,

where the basic $R$ element is a pulse or series of pulses that rotates the spins by an odd number of $\pi$ rotations about the $x$-axis, and $R^{\prime}$ is derived from $R$ by changing the signs of all phases. The subscript $\phi$ denotes the overall RF phase shifts of the $R$ elements and is given ideally by

$\phi=\pi v / N$

while the superscript $N / 2$ denotes the number of repetitions of the bracketed elements making up a complete cycle (in practice, it is usually sufficient to complete an even number of $R$ elements). In the pulse sequences explored in this article, the basic $R$ element is always given by the composite $\pi$ pulse

$R_{0}=R_{0}^{\prime}=90_{0} 270_{180}$

specifying flip angles and phases in degrees. This basic element provides particularly robust performance with respect to chemical shift interactions.

The ${ }^{13} \mathrm{C}$ nutation frequency $\omega_{\text {nut }}^{S}$ is set so that one $R N_{n}^{v}$ sequence occupies exactly $n$ rotor periods. In the case of the $R$ element in Eq. (3), the ${ }^{13} \mathrm{C}$ nutation frequency is set to exactly $N / n$ times the spinning frequency $\omega_{r}$. For example, a $R 12_{2}^{5}$ sequence based on the element in Eq. (3) may be written explicitly as

$\left[90_{75.0} 270_{255.0} 90_{285.0} 270_{105.0}\right]^{6}$,

where the subscripts are the phases of the pulses (in degrees) while the superscript denotes six repetitions of the

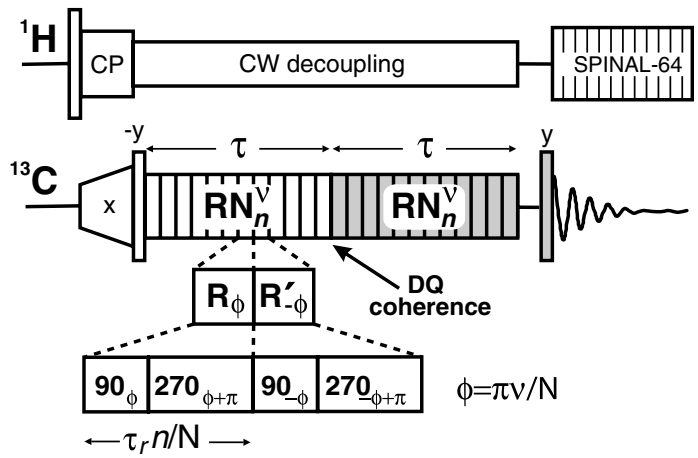

Fig. 2. Pulse sequence diagram for ${ }^{13} \mathrm{C}$ double-quantum homonuclear dipolar recoupling with a symmetry-based $R N_{n}^{v}$ recoupling sequence. bracketed elements. One complete cycle spans two rotor periods and requires an RF field strength corresponding to a nutation frequency which is exactly six times the spinning frequency.

\subsection{Symmetry series}

A symmetry analysis of the sequence $R 12_{2}^{5}$, using the treatment given in $[1,27,42]$ shows that the only homonuclear first-order average Hamiltonian terms $H_{\ell m \lambda \mu}^{(1)}$ which are symmetry-allowed have quantum numbers of the form $\{\ell, m, \lambda, \mu\}=\{2, \pm 1,2, \pm 2\}$ (homonuclear double-quantum terms) or $\{\ell, m, \lambda, \mu\}=\{0,0,0,0\}$ (homonuclear $J$-couplings). Here $\{\ell, m\}$ define the tensorial rank and component index for spatial rotations, while $\{\lambda, \mu\}$ define the tensorial rank and component index for spin rotations, as discussed in [1]. As mentioned above, this pulse sequence requires a ratio of ${ }^{13} \mathrm{C}$ nutation frequency to spinning frequency of $\omega_{\text {nut }}^{S} / \omega_{r}=N / n$, assuming that the basic element in Eq. (3) is used.

The sequence $R 12_{2}^{5}$ is the first member of a series $\left\{R 12_{2}^{5}, R 14_{2}^{6}, R 16_{2}^{7}, R 18_{2}^{8}, \ldots\right\}$ whose elements share the same first-order selection rules, but which have different ${ }^{13} \mathrm{C}$ nutation frequencies $\omega_{\text {nut }}^{S}=N \omega_{r} / n$. It is therefore possible to explore the role of ${ }^{13} \mathrm{C}$ nutation frequency at fixed spinning frequency by running along the series $R N_{n}^{(-1+N / 2)}$ with $N \geqslant 12$. We use this property extensively in the following discussion.

\subsection{Double-quantum filtering}

The $R N_{n}^{v}$ recoupling sequences are incorporated into double-quantum filtered (DQF) experiments as shown in Fig. 2. After ${ }^{1} \mathrm{H} \rightarrow{ }^{13} \mathrm{C}$ ramped cross polarization [45], a $90^{\circ}$ pulse is applied with a phase shift of $-90^{\circ}$ to generate longitudinal ${ }^{13} \mathrm{C}$ magnetization. A recoupling sequence of duration $\tau$ excites DQ coherences which are reconverted into longitudinal magnetization by a second recoupling sequence of the same duration $\tau$. An on-resonance continuous-wave ${ }^{1} \mathrm{H}$ decoupling field with nutation frequency $\omega_{\text {nut }}^{I}$ is applied simultaneously with the ${ }^{13} \mathrm{C}$ recoupling sequence. After a short delay, a final $90^{\circ}$ pulse is applied on the ${ }^{13} \mathrm{C}$ channel and ${ }^{1} \mathrm{H}$ SPINAL-64 decoupling [46] is applied during the acquisition period. The shaded elements are given a four-step phase cycle to select signals passing through DQ coherences.

In the experiments discussed below, the recoupling efficiency is studied as a function of the following parameters: the magic-angle-spinning frequency $\omega_{r}$, the ${ }^{1} \mathrm{H}$ decoupling nutation frequency $\omega_{\text {nut }}^{I}$ during the homonuclear recoupling sequence, the ${ }^{13} \mathrm{C}$ recoupling nutation frequency $\omega_{\text {nut }}^{S}$, and the symmetry number $N$ of the $R N_{n}^{(-1+N / 2)}$ recoupling sequence. The symmetry number $n$ is held fixed at $n=2$. The synchronization requirements of the $R N_{n}^{v}$ sequences requires the 
relationship $\omega_{\text {nut }}^{S}=N \omega_{r} / n$. There are therefore three independent parameters to consider.

\section{Experimental}

\subsection{Measurement of double-quantum-filtering efficiency}

For all of the results presented below, the DQ efficiencies were estimated according to the following procedure. For a given $R N_{n}^{v}$ sequence and spinning frequency $\omega_{r}$, the number of $R$ elements giving the maximum DQF signal was determined. This number was held fixed while the efficiency was optimized by small adjustments to the ${ }^{13} \mathrm{C}$ RF power and $R$ element phase shift $\phi$ (documented in Supplementary Material). Once optimized, a series of ${ }^{13} \mathrm{C}$ DQ filtered spectra were obtained (with four or eight acquisitions each), with the strength of the ${ }^{1} \mathrm{H}$ decoupling during ${ }^{13} \mathrm{C}$ homonuclear recoupling incremented from 0 to $120 \mathrm{kHz}$ in increments of $3 \mathrm{kHz}$. The DQ filtering efficiencies were calculated by comparing the integrals of the DQ filtered spectra to a cross polarization spectrum obtained under identical conditions.

A range of MAS frequencies between 6 and $20 \mathrm{kHz}$ and a series of $R N_{n}^{v}$ sequences with $n=2, N=12,14,16 \ldots$ and $v=-1+N / 2$ were studied. All experiments were performed in a field of $9.4 \mathrm{~T}$ using well-filled $3.2 \mathrm{~mm}$ zirconia rotors on a Varian InfinityPlus console. The ${ }^{1} \mathrm{H}$ nutation frequency during the SPINAL-64 decoupling sequence applied during signal acquisition was fixed at $100 \mathrm{kHz}$. The full details of the experimental parameters used are provided in the Supplementary Material.

\subsection{Samples}

These investigations were carried out three representative organic compounds: diammonium- $\left[2,3-{ }^{13} \mathrm{C}_{2}\right]$ fumarate diluted to $10 \%$ in natural abundance diammonium fumarate (DAF), $\left[{ }^{15} \mathrm{~N},{ }^{13} \mathrm{C}_{2}\right]$ glycine with $99 \%{ }^{15} \mathrm{~N}$ and ${ }^{13} \mathrm{C}$ labelling (Gly), and $\left[2,3-{ }^{13} \mathrm{C}_{2}\right]$ alanine with $99 \%$ ${ }^{13} \mathrm{C}$ labelling (Ala). These compounds broadly represent typical classes of ${ }^{13} \mathrm{C}$ spin systems involving $\mathrm{CH}, \mathrm{CH}_{2}$, and $\mathrm{CH}_{3}$ groups, respectively. Their molecular structures are shown in Fig. 1.

Note that the relatively low labelling level of the DAF sample leads to prominent natural abundance peaks from isolated ${ }^{13} \mathrm{C}$ nuclei (Fig. 1A, left column). These signals disappear after double-quantum filtration (right column).

\section{Results}

\subsection{Diammonium- $\left[2,3-{ }^{13} C_{2}\right]$ fumarate (DAF)}

Fig. 3 presents the effect of the ${ }^{1} \mathrm{H}$ decoupling nutation frequency on the ${ }^{13} \mathrm{C}$ DQ filtering efficiency for
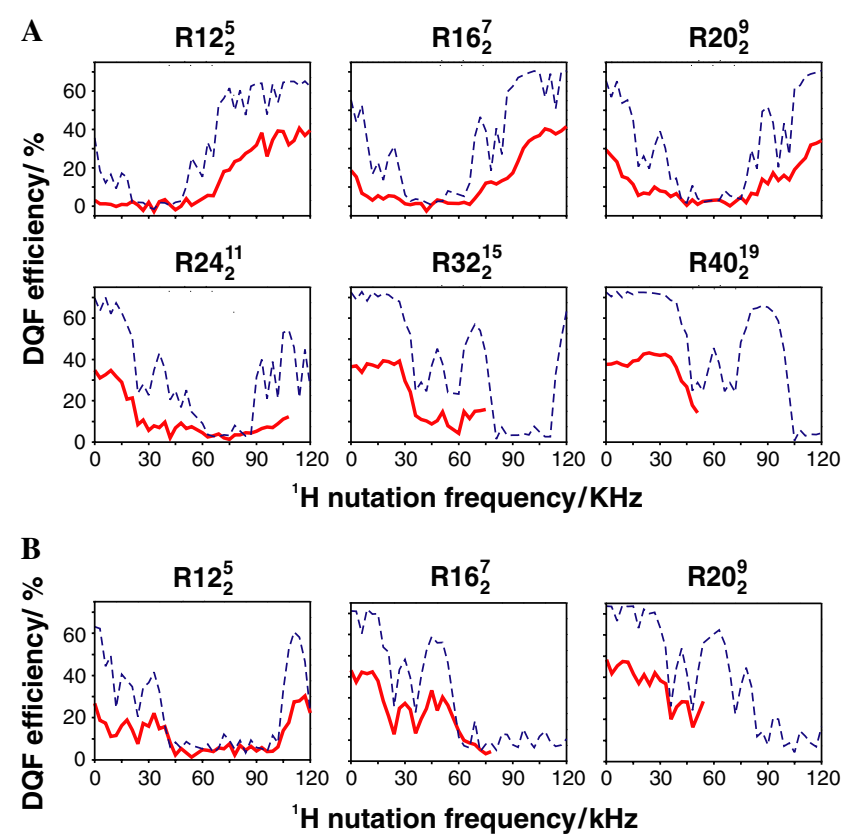

Fig. 3. Plots of ${ }^{13} \mathrm{C}$ double-quantum recoupling efficiency vs. ${ }^{1} \mathrm{H}$ decoupling nutation frequency for DAF with indicated $R N_{n}^{v}$ sequences at (A) $\omega_{r} / 2 \pi=6 \mathrm{kHz}$ and (B) $\omega_{r} / 2 \pi=12 \mathrm{kHz}$. The experimental and simulated data are plotted as solid and dashed lines, respectively. Some of the experimental data sets are incomplete, due to power-handling limitations of the RF probe.

DAF at spinning frequencies of 6 and $12 \mathrm{kHz}$. At $\omega_{r} / 2 \pi=6 \mathrm{kHz}$ (Fig. 3A), the sequence $R N_{n}^{v}$ exhibits the familiar behaviour in which the best DQ filtering efficiency is achieved with a large ${ }^{1} \mathrm{H}$ decoupling nutation frequency of $\omega_{\text {nut }}^{I} / 2 \pi=120 \mathrm{kHz}$, which is approximately three times the ${ }^{13} \mathrm{C}$ nutation frequency used for the recoupling, $\omega_{\text {nut }}^{S} / 2 \pi=36 \mathrm{kHz}$. As the symmetry number $N$ is increased (corresponding to an increase in ${ }^{13} \mathrm{C}$ nutation frequency since $\left.\omega_{\text {nut }}^{S}=N \omega_{r} / n\right)$ the DQ filtering efficiency increases at zero decoupling field, and a dip in the DQ filtering efficiency becomes evident in the vicinity of Hartmann-Hahn match, $\omega_{\text {nut }}^{I}=\omega_{\text {nut }}^{S}$. This minimum shifts to higher $\omega_{\text {nut }}^{I}$ as the $N$ value of the sequence increases. For the sequence with the largest $N\left(R 40_{2}^{19}\right)$, and therefore the largest ${ }^{13} \mathrm{C}$ nutation frequency, the DQ filtering efficiency without ${ }^{1} \mathrm{H}$ decoupling is $37 \%$ and is comparable to the $40 \%$ recoupling efficiency obtained with the lowest $N$ and maximum ${ }^{1} \mathrm{H}$ decoupling power $\left(R 12_{2}^{5}\right)$.

For $\omega_{r} / 2 \pi=12 \mathrm{kHz}$ (Fig. 3B), similar trends in the effects of ${ }^{1} \mathrm{H}$ decoupling on the ${ }^{13} \mathrm{C}$ DQ filtering efficiency are seen. At this increased spinning frequency, periodic local minima in the plots appear, separated by the spinning frequency of $12 \mathrm{kHz}$. A respectable DQ filtering efficiency of $48 \%$ was achieved at $\omega_{r} / 2 \pi=12 \mathrm{kHz}$ using the $R 20_{2}^{9}$ sequence without ${ }^{1} \mathrm{H}$ decoupling.

To display the interaction of ${ }^{1} \mathrm{H}$ and ${ }^{13} \mathrm{C}$ nutation frequencies for the entire series of $R N_{n}^{v}$ sequences, two-dimensional contour plots were constructed. Contour 
plots of experimental DQ filtering efficiencies for DAF at spinning frequencies of 6 and $12 \mathrm{kHz}$ are presented in Figs. 4A and B, respectively. The traces shown in Fig. 3 are horizontal slices through these contoured surfaces.

These contour plots clearly show two regimes for efficient DQ homonuclear dipolar recoupling in the presence of heteronuclear dipolar interactions, separated by a region in which the DQ filtering is poor. The first regime, indicated by the circled number 1 in the plot of Fig. 4A, utilizes strong ${ }^{1} \mathrm{H}$ decoupling to remove the ${ }^{1} \mathrm{H}-{ }^{13} \mathrm{C}$ heteronuclear dipolar interactions while the ${ }^{13} \mathrm{C}$ nutation frequency and the spinning frequency are kept moderate, to avoid Hartmann-Hahn matching. The second regime, indicated by the circled number 2 in the plot of Fig. $4 \mathrm{~A}$, uses strong ${ }^{13} \mathrm{C}$ irradiation to remove the ${ }^{1} \mathrm{H}-{ }^{13} \mathrm{C}$ heteronuclear dipolar interactions. In this regime, good homonuclear recoupling may be achieved without ${ }^{1} \mathrm{H}$ decoupling, as reported for several symmetries of the $C N_{n}^{v}$ type by Hughes et al. [44].

The second regime was explored further at higher MAS frequencies. Fig. 5 displays DQ filtering efficiency as a function of ${ }^{1} \mathrm{H}$ decoupling nutation frequency for DAF using the $R 12_{2}^{5}$ sequence at MAS frequencies from 6 to $20 \mathrm{kHz}$. As the spinning frequency increases, the DQ filtering efficiency increases under no-decoupling conditions. The periodic dips in the DQ filtering efficiency as a function of ${ }^{1} \mathrm{H}$ decoupling nutation frequency become increasingly evident as the spinning frequency is increased. The increase of DQ filtering efficiencies with increasing spinning frequency, under no-decoupling conditions, is shown more clearly in Fig. 6.

\section{2. $\left[{ }^{15} N,{ }^{13} C_{2}\right]$ Glycine (Gly)}

The effects of ${ }^{1} \mathrm{H}$ decoupling during ${ }^{13} \mathrm{C}$ recoupling were also studied for $\left[{ }^{15} \mathrm{~N},{ }^{13} \mathrm{C}_{2}\right]$ glycine for a series of $R N_{n}^{v}$ sequences and MAS frequencies of 6 and $12 \mathrm{kHz}$ (Fig. 4). The appearance of these plots is qualitatively similar to DAF, but the region of poor recoupling is much broader. As discussed below, this is due to the

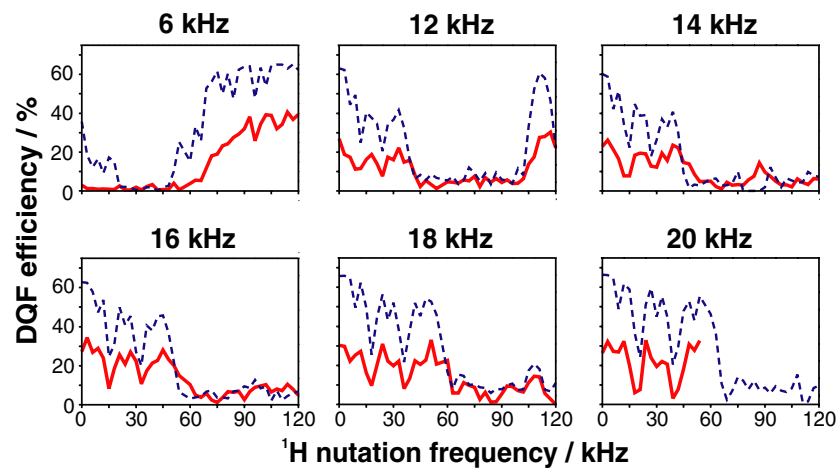

Fig. 5. Double-quantum filtering efficiencies for DAF using the recoupling sequence $R 12_{2}^{5}$ as a function of ${ }^{1} \mathrm{H}$ decoupling nutation frequency, at the indicated MAS frequencies $\left(\omega_{r} / 2 \pi\right)$. The experimental and simulated data are plotted as solid and dashed lines, respectively.
A

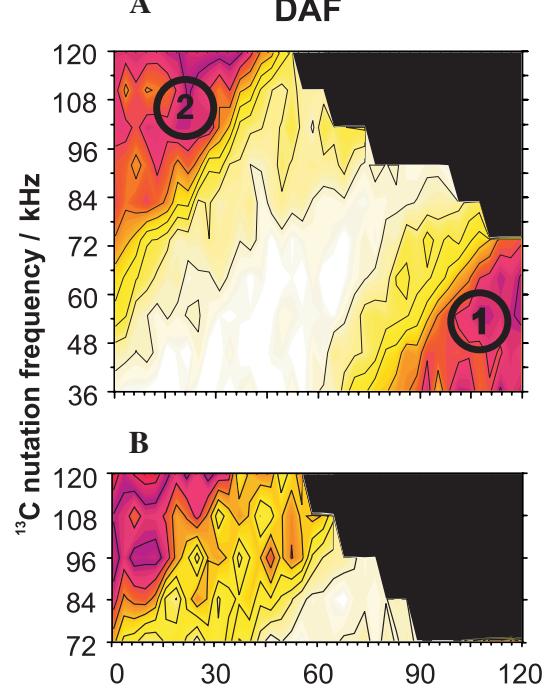

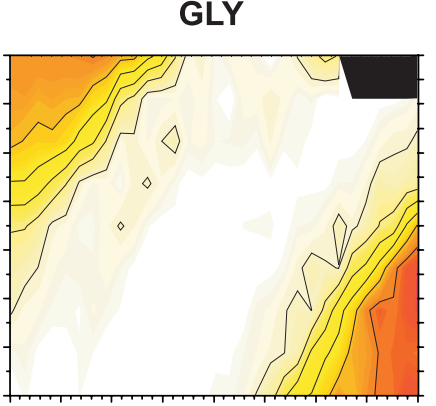

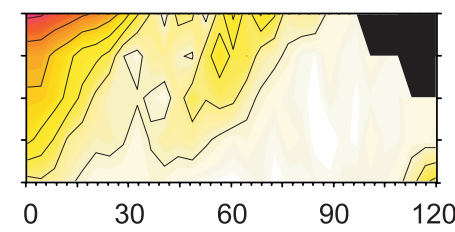

${ }^{1} \mathrm{H}$ nutation frequency $/ \mathrm{kHz}$

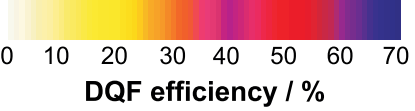

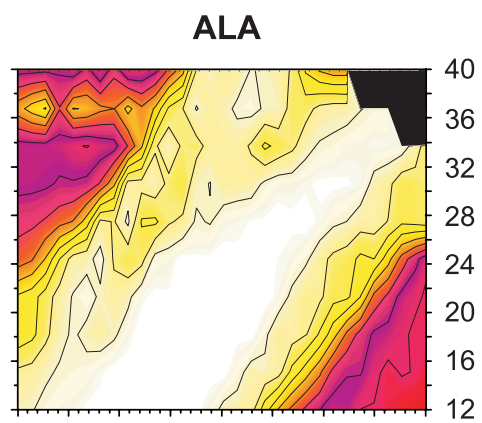

$N$

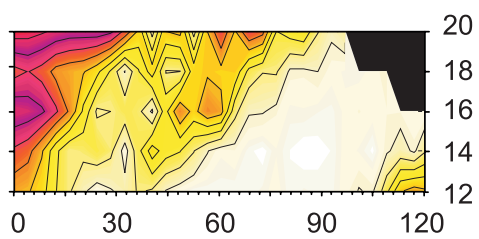

$N$

Fig. 4. Contour plots of the experimental ${ }^{13} \mathrm{C}$ double-quantum filtering efficiency at a spinning frequency of (A) $6 \mathrm{kHz}$ and (B) $12 \mathrm{kHz}$ for DAF, Gly, and Ala. The ${ }^{1} \mathrm{H}$ decoupling nutation frequency $\omega_{\text {nut }}^{I} / 2 \pi$ is plotted along the horizontal axis while the ${ }^{13} \mathrm{C}$ recoupling nutation frequency $\omega_{\text {nut }}^{S} / 2 \pi$ is plotted along the vertical axis. The corresponding symmetry number $N$ is indicated on the right-hand vertical axis. The black regions are inaccessible, due to technical limitations on the probe performance. 


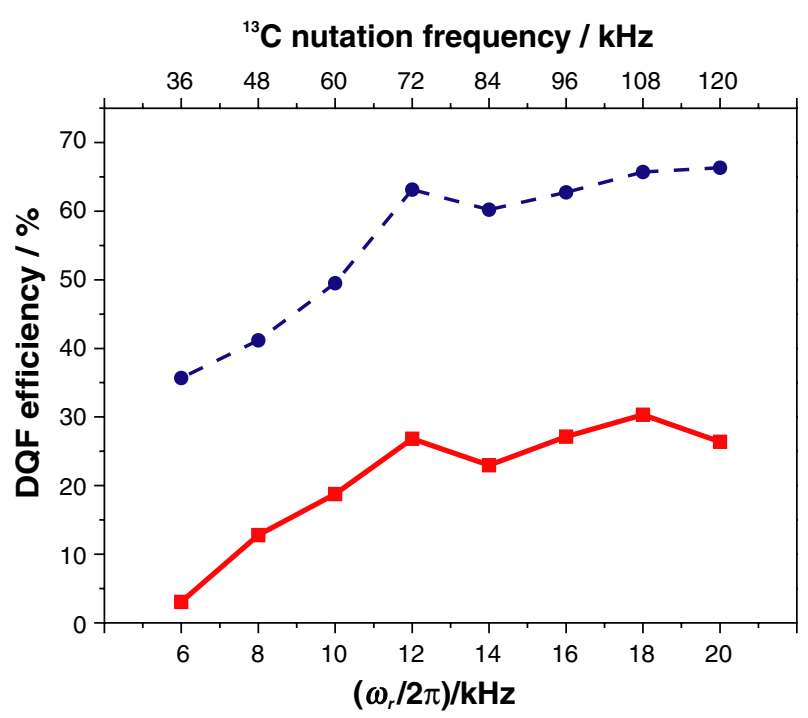

Fig. 6. Double-quantum filtering efficiencies for DAF using the recoupling sequence $R 12_{2}^{5}$, without proton decoupling. The spinning frequency $\omega_{r} / 2 \pi$ is varied along the horizontal axis. The corresponding ${ }^{13} \mathrm{C}$ recoupling nutation frequency is given by $\omega_{\text {nut }}^{S}=6 \omega_{r}$ for all points. The experimental and simulated data are plotted as solid and dashed lines, respectively.

strong ${ }^{1} \mathrm{H}-{ }^{1} \mathrm{H}$ homonuclear interactions in the $\mathrm{CH}_{2}$ group of glycine. Nonetheless, it was still possible to achieve a DQ filtering efficiency of $36 \%$ for glycine using the $R 20_{2}^{9}$ sequence with no ${ }^{1} \mathrm{H}$ decoupling at $12 \mathrm{kHz}$ spinning frequency and $18 \%$ using the $R 12_{2}^{5}$ sequence with no ${ }^{1} \mathrm{H}$ decoupling at $20 \mathrm{kHz}$.

\section{3. $\left[2,3-{ }^{13} C_{2}\right]$ Alanine (Ala)}

The effects of ${ }^{1} \mathrm{H}$ decoupling during ${ }^{13} \mathrm{C}$ recoupling were studied for $\left[2,3-{ }^{13} \mathrm{C}_{2}\right]$ alanine for a series of $R N_{n}^{v}$ sequences and MAS frequencies of 6 and $12 \mathrm{kHz}$ (Fig. 4). These data are similar to the DAF data, probably since the ${ }^{1} \mathrm{H}-{ }^{1} \mathrm{H}$ homonuclear interactions are relatively weak due to the rapid rotation of the $\mathrm{CH}_{3}$ group. It was possible to achieve a DQ filtering efficiency of $48 \%$ for alanine using the $R 20_{2}^{9}$ sequence with no ${ }^{1} \mathrm{H}$ decoupling at $12 \mathrm{kHz}$ spinning frequency and $38 \%$ using the $R 12_{2}^{5}$ sequence with no ${ }^{1} \mathrm{H}$ decoupling at $20 \mathrm{kHz}$.

\section{Simulations}

Simulations of the effects of ${ }^{1} \mathrm{H}$ decoupling on ${ }^{13} \mathrm{C}$ homonuclear recoupling were performed using the SIMPSON platform [47]. The simulations considered the ${ }^{13} \mathrm{C}$ nuclei and their directly-attached ${ }^{1} \mathrm{H}$ nuclei in a single molecule of DAF, Gly, or Ala, ignoring the protons attached to nitrogens, the ${ }^{15} \mathrm{~N}$ nucleus (in the case of Gly) and all intermolecular interactions. Representative SIMPSON input files, containing full details of the simulated spin systems are provided in Supplementary information. For each simulation, the signal intensities were averaged over 100 crystallite orientations. The results of these simulations accompany the experimental data in Figs. 3,5, and 6. The full set of simulations are displayed as contour plots in Fig. 7 to facilitate comparison with the experimental data displayed in Fig. 4.

The simulated efficiencies were typically $20-40 \%$ higher than those observed experimentally. This discrepancy may be attributed to long-range spin-spin interactions, instrumental imperfections (such as phase transients and RF inhomogeneity) and incoherent relaxation. Despite the discrepancy in the overall scale, all the main experimental trends are reproduced qualitatively in the simulations, including the existence of the two regimes, the differences between Gly and the other compounds, and the periodic structures in the plots of DQ filtering efficiency vs. ${ }^{1} \mathrm{H}$ decoupling nutation frequency observed at high spinning frequency.

We have verified by simulation that the large homonuclear coupling between the two $\mathrm{H}^{\alpha}$ protons is responsible for the different behaviour of glycine, compared to DAF and Ala. If the ${ }^{1} \mathrm{H}-{ }^{1} \mathrm{H}$ coupling is removed, the simulated glycine plot is similar to that of DAF (not shown).

\section{Theory}

Hughes et al. [44] addressed the problem of heteronuclear interference with homonuclear recoupling sequences by calculating the magnitude of second-order average Hamiltonian terms, in the absence of a decoupling field. In the discussion below, we show that first-order average Hamiltonian theory provides a sufficient framework for understanding many of the essential features of heteronuclear interference, and allows one to explore the full space of decoupler field variation.

The theory of symmetry-based recoupling in heteronuclear systems has been given in [27]. A slightly modified formulation is required for the case of $\mathrm{CW}$ irradiation of the ${ }^{1} \mathrm{H}$ nuclei. In the following discussion the ${ }^{13} \mathrm{C}$ nuclei are denoted as $S$, while the protons are denoted $I$.

The spin Hamiltonian during the $R N_{n}^{v}$ sequence may be written

$H(t)=H_{S}(t)+H_{I S}(t)+H_{I}(t)$,

where the various terms involve the interactions of the spins with each other, with the molecular environment and with the applied magnetic fields:

$H_{S}(t)=H_{S}^{\mathrm{CS}}(t)+H_{S S}(t)+H_{S}^{\mathrm{RF}}(t)$,

$H_{I}(t)=H_{I}^{\mathrm{CS}}(t)+H_{I I}(t)+H_{I}^{\mathrm{RF}}(t)$,

where $H_{S}^{\mathrm{CS}}(t)$ and $H_{I}^{\mathrm{CS}}(t)$ represent chemical shift interactions, $H_{S S}(t), H_{I S}(t)$ and $H_{I I}(t)$ represent spin-spin couplings, and $H_{S}^{\mathrm{RF}}(t)$ and $H_{I}^{\mathrm{RF}}(t)$ represent interactions 

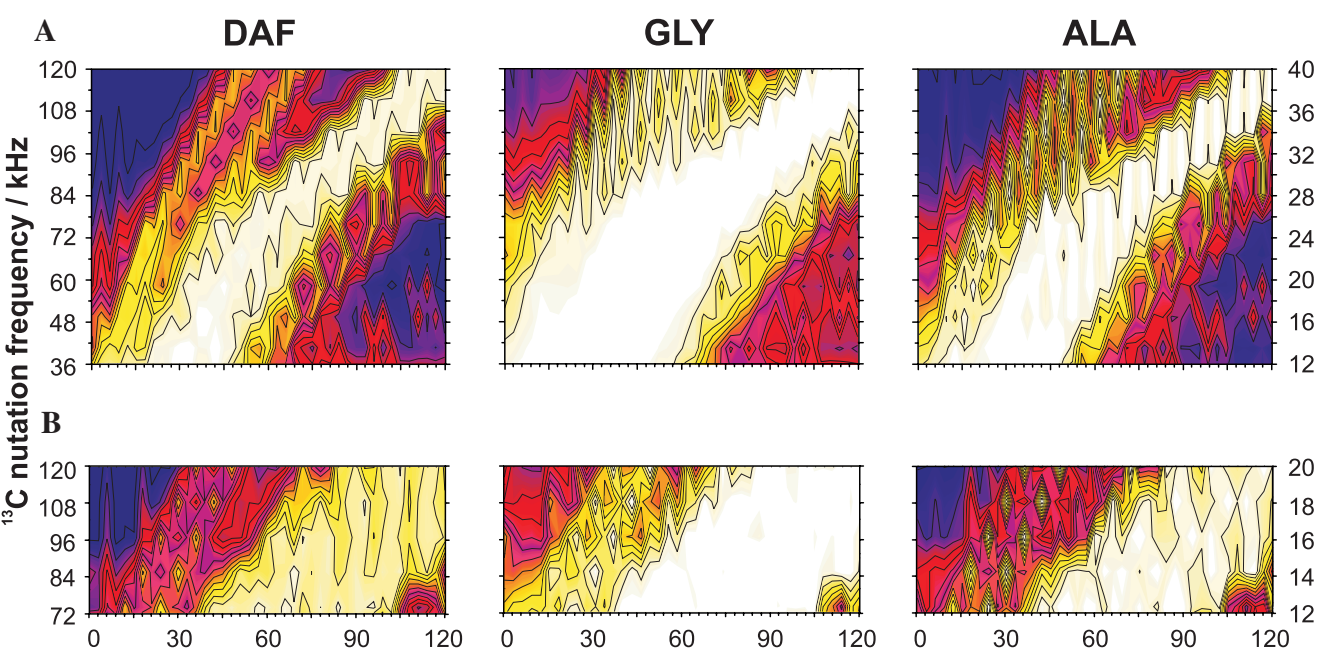

\section{$N$}
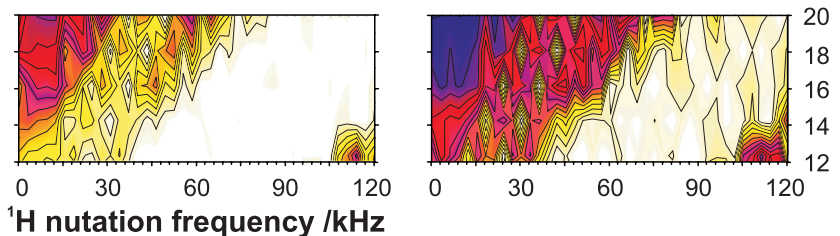

$\boldsymbol{N}$

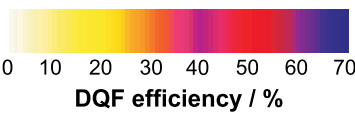

Fig. 7. Contour plots of the simulated ${ }^{13} \mathrm{C}$ double-quantum filtering efficiency at a spinning frequency of (A) $6 \mathrm{kHz}$ and (B) $12 \mathrm{kHz}$ for DAF, Gly, and Ala. The ${ }^{1} \mathrm{H}$ decoupling nutation frequency $\omega_{\text {nut }}^{I} / 2 \pi$ is plotted along the horizontal axis while the ${ }^{13} \mathrm{C}$ recoupling nutation frequency $\omega_{\text {nut }}^{S} / 2 \pi$ is plotted along the vertical axis. The corresponding symmetry number $N$ is indicated on the right-hand vertical axis.

with the resonant RF fields. The spin Hamiltonian terms are time-dependent due to the mechanical sample rotation and the modulation of the applied RF fields. In the case of the $S$-spins, the applied RF field is phasemodulated according to the $R N_{n}^{v}$ scheme

$H_{S}^{\mathrm{RF}}(t)=\omega_{\mathrm{nut}}^{S}\left(S_{x} \cos \phi_{\mathrm{RF}}^{S}(t)+S_{y} \cos \phi_{\mathrm{RF}}^{S}(t)\right)$,

where $\phi_{\mathrm{RF}}^{S}(t)$ is the time-modulated phase of the $S$-spin RF field. For the sequences considered here, the amplitude of the $S$-spin RF field, expressed as a nutation frequency $\omega_{\text {nut }}^{S}=\frac{1}{2}\left|\gamma_{S} B_{\mathrm{RF}}^{S}\right|$, is kept constant.

In the case of the $I$-spins, we consider only $\mathrm{CW}$ irradiation with a constant nutation frequency

$H_{I}^{\mathrm{RF}}=\omega_{\text {nut }}^{I} I_{x}$.

The effect of the RF fields is conveniently described by introducing two RF propagation operators, which obey the equations:

$$
\begin{aligned}
& \frac{\mathrm{d}}{\mathrm{d} t} U_{S}^{\mathrm{RF}}\left(t, t^{0}\right)=-\mathrm{i} H_{S}^{\mathrm{RF}}(t) U_{S}^{\mathrm{RF}}\left(t, t^{0}\right), \\
& \frac{\mathrm{d}}{\mathrm{d} t} U_{I}^{\mathrm{RF}}\left(t, t^{0}\right)=-\mathrm{i} H_{I}^{\mathrm{RF}}(t) U_{I}^{\mathrm{RF}}\left(t, t^{0}\right),
\end{aligned}
$$

where $t^{0}$ is the time point at the start of the recoupling sequence. For the $I$-spins, this propagator has the simple form of a rotation around the $x$-axis of the rotating reference frame

$U_{I}^{\mathrm{RF}}\left(t, t^{0}\right)=R_{x}^{I}\left(\omega_{\text {nut }}^{I}\left(t-t^{0}\right)\right)$,

where

$R_{x}^{I}(\beta)=\exp \left\{-\mathrm{i} \beta I_{x}\right\}$.
For the $S$-spins, the propagator under the modulated $\mathrm{RF}$ field is much more complicated. It may be written in general as

$$
\begin{aligned}
U_{\mathrm{RF}}^{S}\left(t, t^{0}\right)= & R_{z}^{S}\left(\alpha_{\mathrm{RF}}^{S}(t)\right) R_{y}^{S}\left(\beta_{\mathrm{RF}}^{S}(t)\right) R_{z}^{S}\left(\gamma_{\mathrm{RF}}^{S}(t)\right) \\
= & \exp \left\{-\mathrm{i} \alpha_{\mathrm{RF}}^{S}(t) S_{z}\right\} \exp \left\{-\mathrm{i} \beta_{\mathrm{RF}}^{S}(t) S_{y}\right\} \\
& \times \exp \left\{-\mathrm{i} \gamma_{\mathrm{RF}}^{S}(t) S_{z}\right\},
\end{aligned}
$$

where $\left\{\alpha_{\mathrm{RF}}^{S}, \beta_{\mathrm{RF}}^{S}, \gamma_{\mathrm{RF}}^{S}\right\}$ are the three Euler angles describing the rotation induced by the modulated RF field.

It proves convenient to express the $I$-spin rotation in an analogous form to the $S$-spin rotation. If an additional $-\pi / 2$ rotation of the $I$-spins is introduced (equivalent to a transformation into a tilted reference frame), we may write

$U_{\mathrm{RF}}^{I}\left(t, t^{0}\right) R_{y}^{I}(-\pi / 2)=R_{z}^{I}\left(\alpha_{\mathrm{RF}}^{I}\right) R_{y}^{I}\left(\beta_{\mathrm{RF}}^{I}\right) R_{z}^{I}\left(\gamma_{\mathrm{RF}}^{I}(t)\right)$,

where the $I$-spin Euler angles are given by:

$\alpha_{\mathrm{RF}}^{I}=0$,

$\beta_{\mathrm{RF}}^{I}=-\frac{\pi}{2}$,

$\gamma_{\mathrm{RF}}^{I}(t)=-\omega_{\mathrm{nut}}^{I}\left(t-t^{0}\right)$.

The $R N_{n}^{v}$ sequence is designed to impose a periodic time-translation symmetry on the $S$-spin RF Euler angles. As described in [27], this time-translation symmetry has the form:

$\beta_{\mathrm{RF}}^{S}\left(t+q \tau_{R}\right)=\beta_{\mathrm{RF}}^{S}(t)+q \pi$,

$\gamma_{\mathrm{RF}}^{S}\left(t+q \tau_{R}\right)=\gamma_{\mathrm{RF}}^{S}(t)-\frac{2 \pi v_{S}}{N} q$, 
where $\tau_{R}=n \tau_{r} / N$ is the duration of one $R$ element and $q$ is an integer. From now on, the winding number of the $R N_{n}^{v}$ sequence is written as $v_{S}$, for reasons which will shortly become clear.

From Eq. (14), the $I$-spin RF Euler angles obey the equation:

$\beta_{\mathrm{RF}}^{I}\left(t+q \tau_{R}\right)=\beta_{\mathrm{RF}}^{S}(t)$,

$\gamma_{\mathrm{RF}}^{I}\left(t+q \tau_{R}\right)=\gamma_{\mathrm{RF}}^{I}(t)-\omega_{\mathrm{nut}}^{I} q \tau_{R}$.

Now suppose that the $I$-spin nutation frequency is given by

$\omega_{\mathrm{nut}}^{I}=\frac{v_{I} \omega_{r}}{n}$,

where $v_{I}$ is an integer. In this case, the symmetry relationship Eq. (16) may be written:

$\beta_{\mathrm{RF}}^{I}\left(t+q \tau_{R}\right)=\beta_{\mathrm{RF}}^{S}(t)$,

$\gamma_{\mathrm{RF}}^{I}\left(t+q \tau_{R}\right)=\gamma_{\mathrm{RF}}^{I}(t)-\frac{2 \pi v_{I}}{N} q$,

which has a similar form to Eq. (15) but is of the $C N_{n}^{v}$ class of symmetries [1]. The combination of the timetranslation symmetries in Eqs. (15) and (18) therefore define a dual $C R N_{n}^{v_{I}, v_{S}}$ sequence on the $I S$ spin system [27].

The symmetry theory for dual $C R N_{n}^{v_{l}, v_{S}}$ sequences, as described in [27], may therefore be applied directly to the problem of $R N_{n}^{v}$ recoupling in the presence of CW heteronuclear decoupling, providing that the decoupling nutation frequency is such that Eq. (17) is fulfilled for an integer winding number $v_{I}$, and that the additional $\pi / 2$ rotation given in Eq. (13) is taken into account. In principle, RF fields which do not conform to Eq. (17) may be analyzed by splitting the $I$-spin RF field term into two parts: a large part which conforms to Eq. (17), and a smaller perturbation term which is incorporated into the average Hamiltonian theory. In this section, we avoid this complication by assuming that Eq. (17) is exactly fulfilled for an integer winding number $v_{I}$.

The $C R N_{n}^{v_{I}, v_{S}}$ symmetry of the recoupling pulse sequence in the presence of $\mathrm{CW}$ proton decoupling leads directly to the following selection rule for the first-order average Hamiltonian terms [27]:

$\bar{H}_{\ell, m, \lambda_{I}, \mu_{I}, \lambda_{S}, \mu_{S}}^{(1)}=0 \quad$ if $\quad m n-\mu_{I} v_{I}-\mu_{S} v_{S} \neq \frac{N}{2} Z_{\lambda_{S}}$,

where a general spin interaction is defined by the quantum numbers $\left\{\ell, m, \lambda_{I}, \mu_{I}, \lambda_{S}, \mu_{S}\right\}$, representing the rank and component index under spatial rotations, the rank and component index under rotations of the $I$-spins, and the rank and component index under rotations of the $S$-spins, respectively. The symbol $Z_{\lambda_{S}}$ represents any integer with the same parity as the $S$-spin rank $\lambda_{S}$. If $\lambda_{S}$ is odd, then $Z_{\lambda_{S}}$ takes values $\pm 1, \pm 3, \ldots \pm 5$, while if $\lambda_{S}$ is even, then $Z_{\lambda_{S}}$ takes values $0, \pm 2, \ldots \pm 4$.

As an example, consider a $R 14_{2}^{6}$ sequence applied in the presence of a $\mathrm{CW}$ heteronuclear decoupling with a strength corresponding to $\omega_{\text {nut }}^{I}=\frac{15}{2} \omega_{r}$. This is very close to the Hartmann-Hahn match condition, which is expected to be damaging for the homonuclear recoupling. From Eq. (17), this $I$-spin RF field corresponds to a $I$-spin winding number $v_{I}=15$. The selection rule Eq. (19) shows that a first-order average Hamiltonian term vanishes unless the equality $2 m-15 \mu_{I}-6 \mu_{S}=7 Z_{\lambda_{S}}$ is satisfied for an integer $Z_{\lambda_{S}}$ with the same parity as $\lambda_{S}$. The double-quantum homonuclear recoupling properties of the sequence $R 14_{2}^{6}$ follow from the fact that the terms $\left\{\ell, m, \lambda_{I}, \mu_{I}, \lambda_{S}\right.$, $\left.\mu_{S}\right\}=\{2, \pm 1,0,0,2, \mp 2\}$ are symmetry-allowed since $2( \pm 1)-15(0)-6(\mp 2)=7( \pm 2)$. At the same time, the heteronuclear dipole-dipole coupling terms $\left\{\ell, m, \lambda_{I}\right.$, $\left.\mu_{I}, \lambda_{S}, \mu_{S}\right\}=\{2, \pm 1,1, \pm 1, \mp 1\}$ are symmetry-allowed since $2( \pm 1)-15( \pm 1)-6(\mp 1)=7(\mp 1)$. It follows that several heteronuclear terms are symmetry-allowed at the $I$-spin nutation frequency $\omega_{\text {nut }}^{I}=\frac{15}{2} \omega_{r}$, and that this condition should therefore be avoided when applying $\mathrm{CW}$ proton decoupling during $R 14_{2}^{6}$ recoupling.

The selection rule Eq. (19) allows logging of the symmetry-allowed first-order average Hamiltonian terms as a function of the symmetry numbers $\left\{N, n, v_{I}, v_{S}\right\}$, providing that the $I$-spin nutation frequency is an integer multiple of $\omega_{r} / n$. In addition, the magnitude of the symmetry-allowed heteronuclear dipolar coupling terms may be assessed, through the scaling factors $\kappa_{2, m, 1, \mu_{I}, 1, \mu_{S}}^{I S}$, which may be calculated using the method described in [27]. In general, there are several symmetry-allowed heteronuclear terms for each combination of symmetry numbers $\left\{N, n, v_{I}, v_{S}\right\}$. In this case, a qualitative heteronuclear interference factor may be assessed by taking the root sum square of the symmetry-allowed scaling factors

$$
\begin{aligned}
\mathfrak{R} & =\mathfrak{R}\left(N, n, v_{I}, v_{S}\right) \\
& =\left[\sum_{\substack{\left\{m, \mu_{I}, \mu_{S}\right\} \\
\text { symm allowed }}}\left|\kappa_{2, m, 1, \mu_{I}, 1, \mu_{S}}\left(C R N_{n}^{v_{I}, v_{S}}\right)\right|^{2}\right]^{1 / 2},
\end{aligned}
$$

where the sum is taken over all symmetry-allowed combinations of quantum numbers $\left\{m, \mu_{I}, \mu_{S}\right\}$ deriving from a heteronuclear dipole-dipole interaction.

An example of the Mathematica code used for these calculations is given in the Supplementary information.

Although Eq. (18) defines $C N_{n}^{v}$ symmetry for the $I$ spins (in the tilted frame defined by Eq. (13)), the CW irradiation does not have the form of a series of phase-shifted elements, normally used to construct sequences with this symmetry. This illustrates an important point: the mathematical symmetry properties of the interaction frame Hamiltonian are more general than the usual construction principles for these symmetries. In other words, the usual construction principles of $C N_{n}^{v}$ and $R N_{n}^{v}$ sequences are sufficient, but not necessary, to generate the $C N_{n}^{v}$ and $R N_{n}^{v}$ selection rules. In the 
present case, $C N_{n}^{v}$ symmetry is generated in a tilted frame by an unmodulated RF field.

Fig. 8 plots the heteronuclear interference factor $\mathfrak{R}\left(N, n, v_{I}, v_{S}\right)$ for a parameter space corresponding to the simulations of DQ filtered efficiency shown in Fig. 7A. As in those simulations, the condition $n=2$ is kept fixed, while $N$ and $v_{S}$ are varied simultaneously along the vertical axis, according to $v_{S}=\frac{1}{2} N-1$, so as to maintain the homonuclear double-quantum recoupling condition. To assist comparison with Fig. 7A, the top horizontal axis has been labelled according to the $I$-spin nutation frequency corresponding to the plotted values of $v_{I}$, at the same spinning frequency $\omega_{r} / 2 \pi=6 \mathrm{kHz}$ used in the simulations. The right-hand vertical axis has been labelled with the $S$-spin nutation frequency corresponding to the plotted values of $N$ and $v_{S}$, under the same conditions.

There is a striking qualitative resemblance between the plots in Figs. 7A and 8. Conditions providing a large value of $\mathfrak{R}$ correspond very well to regions of poor DQ filtering performance. The two regimes under which good DQ filtering performance is observed correspond to regions free from points displaying large values of $\Re$. The broad, roughly diagonal, band of very poor DQ filtering efficiency in Fig. 7A corresponds closely to a similar band of finite $\mathfrak{R}$ values in Fig. 8 .

A closer examination of the two plots does, however, reveal some discrepancies. The symmetry analysis predicts that a small change in $I$-spin nutation frequency by one-half of the spinning frequency should radically change the degree of heteronuclear interference. Although simulations do agree with this prediction in

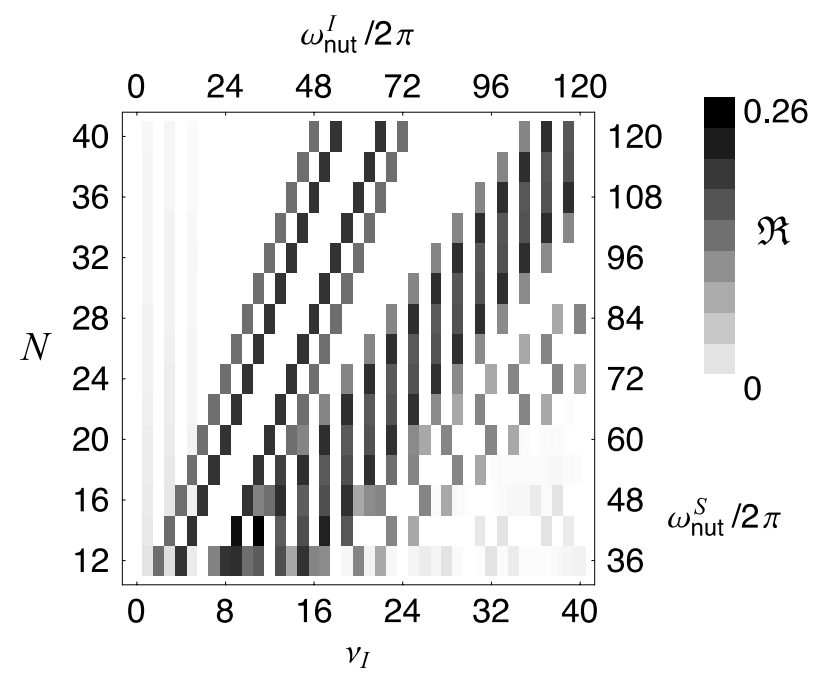

Fig. 8. Heteronuclear recoupling factor $\mathfrak{R}$, as defined in Eq. (20), plotted as a function of the symmetry numbers $v_{I}$ (horizontal axis) and $N$ (vertical axis), for the set of sequences $C R N_{n}^{v_{I}, v_{S}}$, with $n=2$ and $v_{S}=-1+N / 2$. The chosen parameter space corresponds to the simulation conditions in Fig. 7A, with which this plot should be compared. The corresponding nutation frequencies at a spinning frequency of $6 \mathrm{kHz}$ are indicated at the top and right edges of the plot. some regions of the plot, they do not confirm the predicted sensitivity to the $I$-spin nutation frequency in other regions - especially near the broad, near-diagonal, band of very poor DQ filtering efficiency, where the DQ filtering efficiency appears to be insensitive to small changes in $\omega_{\text {nut }}^{I}$. In addition, the first-order average Hamiltonian analysis cannot explain the qualitatively different behaviour of glycine, which is due to the strong homonuclear interactions in that compound.

Brinkmann and Edén [49] showed that the magnitude of second-order average Hamiltonian terms is often increased in the vicinity of conditions which lead to finite first-order terms. A region of parameter space which has a high density of finite first-order terms is therefore likely to provide considerable second-order terms, even if the first-order terms disappear under particular conditions. This effect is probably sufficient to explain the qualitative appearance of the simulated plots, including the expanded region of poorer performance for glycine. The experimental plots of double-quantum-filtering efficiency will be further broadened by effects such as RF inhomogeneity.

We have also calculated heteronuclear interference factors for homonuclear recoupling sequences of the type $C N_{2}^{1}$, where $7 \leqslant N \leqslant 20$ (see Supplementary Information). The first-order average Hamiltonian theory predicts a very similar behaviour to the corresponding set of $R$-sequences.

\section{Discussion}

The main conclusions of this study are that there are two experimental regimes under which heteronuclear interference with $R N_{n}^{v}$ sequences is minimized. In the first regime, the $S$-spin RF field is moderated, while the $I$-spin $\mathrm{RF}$ field is as strong as possible. This regime is usually only suitable for low spinning frequencies. In the second regime, the $S$-spin RF field is as strong as possible, while the $I$-spin RF field is weak or absent. This regime allows good recoupling performance at moderate to high spinning frequencies. The second regime is a better match to the typical experimental conditions for most current solid-state NMR experiments, and is expected to become popular. As an example, we have successfully performed double-quantum experiments on ${ }^{13} \mathrm{C}_{2}$-labelled rhodopsin samples at a spinning frequency of $10 \mathrm{kHz}$, using a $R 20_{2}^{9}$ sequence without proton decoupling [48].

Although the two regimes are observed for a variety of samples, the details of the behaviour are sample-dependent. As usual $\mathrm{CH}_{2}$ groups present the most severe challenges for ${ }^{13} \mathrm{C}$ recoupling experiments, due to the strong heteronuclear ${ }^{1} \mathrm{H}-{ }^{13} \mathrm{C}$ and homonuclear ${ }^{1} \mathrm{H}-{ }^{1} \mathrm{H}$ couplings. For substances like glycine, the no-decoupling regime is only accessible when the strongest $S$-spin RF fields are used. 
We have also performed preliminary investigations of ${ }^{13} \mathrm{C}-{ }^{13} \mathrm{C}$ recoupling over longer distances, where supercycles are necessary to stabilize the long-term performance [40]. The effects of heteronuclear interference are similar to those observed at short range.

In summary, we have confirmed the conclusions of Hughes et al. [44], showing that symmetry-based recoupling sequences produce good homonuclear recoupling without heteronuclear decoupling, even at low or moderate spinning frequencies. We have extended the study of Hughes et al. [44] to a range of $R N_{n}^{v}$ sequences, exploring a wide range of nutation frequencies on both spin species. A first-order average Hamiltonian analysis describes the simulated and experimental spin dynamics rather well. A more quantitative understanding of these phenomena may require simulations of larger numbers of spins, and the inclusion of effects such as RF inhomogeneity.

\section{Acknowledgments}

This research was supported by the EPSRC (UK). D.H.B. was supported by the Natural Sciences and Engineering Research Council of Canada. We thank O.G. Johannessen for instrumental support and A. Sebald for providing the ${ }^{13} \mathrm{C}$-labelled diammonium fumarate sample.

\section{Appendix A. Supplementary data}

Supplementary data associated with this article can be found, in the online version, at doi:10.1016/ j.jmr.2005.07.020. Full-colour versions of the plots may be viewed in the on-line version.

\section{References}

[1] M.H. Levitt, Symmetry-based pulse sequences in magic-angle spinning solid-state NMR, in: D.M. Grant, R.K. Harris (Eds.), Encyclopedia of Nuclear Magnetic Resonance: Supplementary Volume, Wiley, Chichester, UK, 2002, pp. 165-196.

[2] Y.K. Lee, N.D. Kurur, M. Helmle, O.G. Johannessen, N.C. Nielsen, M.H. Levitt, Efficient dipolar recoupling in the NMR of rotating solids. A sevenfold symmetric radiofreqency pulse sequence, Chem. Phys. Lett. 242 (1995) 304.

[3] X. Feng, Y.K. Lee, D. Sandström, M. Edén, H. Maisel, A. Sebald, M.H. Levitt, Direct determination of a molecular torsional angle by solid-state NMR, Chem. Phys. Lett. 257 (1996) 314.

[4] W.A. Dollase, M. Feike, H. Förster, T. Schaller, I. Schnell, A. Sebald, S. Steuernagel, A 2D P-31 MAS NMR study of polycrystalline Cd-3(PO4)(2), J. Am. Chem. Soc. 119 (1997) 3807.

[5] X. Feng, P.J.E. Verdegem, Y.K. Lee, D. Sandström, M. Edén, P. Bovée-Geurts, W.J. de Grip, J. Lugtenburg, H.J.M. de Groot, M.H. Levitt, Direct determination of a molecular torsional angle in the membrane protein Rhodopsin by Solid-State NMR, J. Am. Chem. Soc. 119 (1997) 6853.

[6] X. Feng, M. Edén, A. Brinkman, H. Luthman, L. Eriksson, A. Gräslund, O.N. Antzutkin, M.H. Levitt, Direct determination of a peptide torsional angle by Solid-State NMR, J. Am. Chem. Soc. 119 (1997) 12006.

[7] M. Hohwy, H.J. Jakobsen, M. Edén, M.H. Levitt, N.C. Nielsen, Broadband dipolar recoupling in the nuclear magnetic resonance of rotating solids: a compensated $\mathrm{C} 7$ pulse sequence, J. Chem. Phys. 108 (1998) 2686.

[8] Y. Ishii, J. Ashida, T. Terao, ${ }^{13} \mathrm{C}-{ }^{1} \mathrm{H}$ dipolar recoupling dynamics in ${ }^{13} \mathrm{C}$ multiple pulse solid-state NMR, Chem. Phys. Lett. 246 (1995) 439-445.

[9] C.M. Rienstra, M.E. Hatcher, L.J. Mueller, B. Sun, S.W. Fesik, R.G. Griffin, Efficient multispin homonuclear double-quantum recoupling for magic-angle spinning NMR: ${ }^{13} \mathrm{C}-{ }^{13} \mathrm{C}$ correlation spectrscopy of U-13C-Erythromycin A, J. Am. Chem. Soc. 120 (1998) 10602-10612.

[10] A.E. Bennett, C.M. Rienstra, J.M. Griffiths, W. Zhen, P.T. Lansbury, R.G. Griffin, Homonuclear radio frequency-driven recoupling in rotating solids, J. Chem. Phys. 108 (1998) 94639479.

[11] R. Witter, P. Hartmann, J. Vogel, C. Jäger, Measurements of chain length distributions in calcium phosphate glasses using 2D P-31 double quantum NMR, Sol. State Nucl. Magn. Reson. 13 (1998) 189-200.

[12] M. Edén, M.H. Levitt, Pulse sequence symmetries in the nuclear magnetic resonance of spinning solids: Application to heteronuclear decoupling, J. Chem. Phys. 111 (1999) 1511-1519.

[13] M. Edén, M.H. Levitt, Excitation of carbon-13 triple quantum coherence in magic-angle-spinning NMR, Chem. Phys. Lett. 293 (1998) 173.

[14] F. Fayon, C. Bessada, J.P. Coutures, D. Massiot, High-resolution double-quantum P-31 MAS NMR study of the intermediaterange order in crystalline and glass lead phosphates, Inorg. Chem. 38 (1999) 5212-5218.

[15] H. Geen, R. Graf, A.S.D. Heinrichs, B.S. Hickman, I. Schnell, H.W. Spiess, J.J. Titman, Spin counting with fast MAS, J. Magn. Reson. 138 (1999) 167.

[16] M. Hohwy, C.M. Rienstra, C.P. Jaroniec, R.G. Griffin, Fivefold symmetric homonuclear dipolar recoupling in rotating solids: Application to double quantum spectroscopy, J. Chem. Phys. 110 (1999) 7983-7992.

[17] A.N. Appleyard, R.B. Herbert, P.J.F. Henderson, A. Watts, P.J.R. Spooner, Selective NMR observation of inhibitor and sugar binding to the galactose- $\mathrm{H}^{+}$symport protein GalP, of Escherichia coli, Biochim. Biophys. Acta 1509 (2000) 55-64.

[18] A. Brinkmann, M. Edén, M.H. Levitt, Synchronous helical pulse sequences in magic-angle spinning nuclear magnetic resonance: Double quantum recoupling of multiple-spin systems, J. Chem. Phys. 112 (2000) 8539-8554.

[19] M. Carravetta, M. Edén, X. Zhao, A. Brinkmann, M.H. Levitt, Symmetry principles for the design of radiofrequency pulse sequences in the nuclear magnetic resonance of rotating solids, Chem. Phys. Lett. 321 (2000) 205-215.

[20] M. Edén, A. Brinkmann, H. Luthman, L. Eriksson, M.H. Levitt, Determination of molecular geometry by high-order multiplequantum evolution in solid-state NMR, J. Magn. Reson. 144 (2000) 266-279.

[21] S.D. Heinrichs, H. Geen, J.J. Titman, MAS double-quantum filtered dipolar shift correlation spectroscopy, J. Magn. Reson. 147 (2000) 68-77.

[22] X. Helluy, C. Marichal, A. Sebald, Through-bond indirect and through-space direct dipolar coupling P-31 MAS NMR constraints for spectral assignment in the cubic $3 \times 3 \times 3$ superstructure of Ti2O7, J. Phys. Chem. B 104 (2000) 2836-2845.

[23] A. Le Sauze, L. Montagne, G. Palavit, F. Fayon, R. Marchand, X-ray photoelectron spectroscopy and nuclear magnetic resonance structural study of phosphorus oxynitride glasses, 'LiNaPON', J. Non Cryst. Solids 263 (2000) 139145 . 
[24] S. Ravindranathan, X. Feng, T. Karlsson, G. Widmalm, M.H. Levitt, Investigation of carbohydrate conformation in solution and in powders by double-quantum NMR, J. Am. Chem. Soc. 122 (2000) 1102-1115.

[25] D.F. Schantz, J. Schmedt auf der Günne, H. Koller, R.F. Lobo, Multiple-quantum H-1 MAS NMR studies of defect sites in asmade all-silica ZSM-12 zeolite, J. Am. Chem. Soc. 122 (2000) 6659-6663.

[26] M. Carravetta, M. Edén, O.G. Johannessen, H. Luthman, P.J.E. Verdegem, J. Lugtenburg, A. Sebald, M.H. Levitt, Estimation of carbon-carbon bond lengths and medium range internuclear distances by solid-state nuclear magnetic resonance, J. Am. Chem. Soc. 123 (2001) 10628-10638.

[27] A. Brinkmann, M.H. Levitt, Symmetry principles in the nuclear magnetic resonance of spinning solids: heteronuclear recoupling by generalized Hartmann-Hahn sequences, J. Chem. Phys. 115 (2001) 357-384.

[28] J.C.C. Chan, C-REDOR: rotational echo double resonance under very fast magic-angle spinning, Chem. Phys. Lett. 335 (2001) 289297.

[29] J.C.C. Chan, G. Brunklaus, R sequences for the scalar-coupling mediated homonuclear correlation spectroscopy under fast magicangle spinning, Chem. Phys. Lett. 349 (2001) 104-112.

[30] P.K. Madhu, X. Zhao, M.H. Levitt, High-resolution H-1 NMR in the solid state using symmetry-based pulse sequences, Chem. Phys. Lett. 346 (2001) 142-148.

[31] S. Ravindranathan, T. Karlsson, K. Lycknert, G. Widmalm, M.H. Levitt, Conformation of the glycosidic linkage in a disaccharide investigated by double-quantum solid-state NMR, J. Magn. Reson. 151 (2001) 136-141.

[32] X. Zhao, J.L. Sudmeier, W.W. Bachovchin, M.H. Levitt, Measurement of $\mathrm{NH}$ bond lengths by fast magic-angle spinning solidstate NMR spectroscopy: a new method for the quantification of hydrogen bonds, J. Am. Chem. Soc. 123 (2001) 11097-11098.

[33] M. Baldus, Correlation experiments for assignment and structure elucidation of immobilized polypeptides under magic angle spinning, Prog. NMR Spectrosc. 41 (2002) 1-151.

[34] A. Brinkmann, J. Schmedt auf der Günne, M.H. Levitt, Homonuclear zero-quantum recoupling in fast magic-angle spinning nuclear magnetic resonance, J. Magn. Reson. 56 (2002) 79-96.

[35] P.E. Kristiansen, D.J. Mitchell, J.N.S. Evans, Double-quantum dipolar recoupling at high magic-angle spinning rates, J. Magn. Reson. 157 (2002) 253-266.

[36] C.E. Hughes, J. Schmedt auf der Günne, M.H. Levitt, A test for the number of coupled spins $I=1 / 2$ in magic-angle-spinning solids: zero-quantum recoupling of multiple-quantum coherences, ChemPhysChem 4 (2003) 457-465.

[37] J. Schmedt auf der Günne, Distance measurements in spin-1/2 systems by C-13 and P-31 solid-state NMR in dense dipolar networks, J. Magn. Reson. 165 (2003) 18-32.
[38] M. Carravetta, Y. Murata, M. Murata, I. Heinmaa, R. Stern, A. Tontcheva, A. Samoson, Y. Rubin, K. Komatsu, M.H. Levitt, Solid-state NMR spectroscopy of molecular hydrogen trapped inside an open-cage fullerene, J. Am. Chem. Soc. 126 (2004) 40924093.

[39] M. Carravetta, X. Zhao, O.G. Johannessen, W.C. Lai, M.A. Verhoeven, P.H.M. Bovee-Geurts, P.J.E. Verdegem, S. Kiihne, H. Luthman, H.J.M. de Groot, W.J. de Grip, J. Lugtenburg, M.H. Levitt, Protein-induced bonding perturbation of the rhodopsin chromophore detected by double-quantum solid-state NMR, J. Am. Chem. Soc. 126 (2004) 3948-3953.

[40] P.E. Kristiansen, M. Carravetta, W.C. Lai, M.H. Levitt, A robust pulse sequence for the determination of small homonuclear dipolar couplings in magic-angle spinning NMR, Chem. Phys. Lett. 390 (2004) 1-7.

[41] M. Edén, Order-selective multiple-quantum excitation in magic-angle spinning NMR: creating triple-quantum coherences with a trilinear Hamiltonian, Chem. Phys. Lett. 366 (2002) 469-476.

[42] M. Edén, A. Brinkmann, Triple-quantum dynamics in multiplespin systems undergoing magic-angle spinning: application to C13 homonuclear correlation spectroscopy, J. Magn. Reson. 173 (2005) 259-279.

[43] Y. Ishii, 13C-13C dipolar recoupling under vary fast magic-angle spinning in solid-state nuclear magnetic resonance: applications to distance measurements, spectral assignments, and high-throughput secondary-structure determination, J. Chem. Phys. 114 (2001) 8473-8483.

[44] C.E. Hughes, S. Luca, M. Baldus, Radio-frequency driven polarization transer without heteronuclear decoupling in rotating solids, Chem. Phys. Lett. 384 (2004) 435-440.

[45] G. Metz, X. Wu, S.O. Smith, Ramped-amplitude cross-polarization in magic-angle spinning NMR, J. Magn. Reson. A 110 (1994) 219.

[46] B.M. Fung, A.K. Khitrin, K. Ermolaev, An improved broadband decoupling sequence for liquid crystals and solids, J. Magn. Reson. 142 (2000) 97-101.

[47] M. Bak, J.T. Rasmussen, N.C. Nielsen, SIMPSON: a general simulation program for solid state NMR, J. Magn. Reson. 147 (2000) 296-330.

[48] W.C. Lai, N.J. McLean, M.A. Verhoeven, A. Gansmüller, P.H.M. Bovee-Geurts, M. Carravetta, O.G. Johannessen, R.C.D. Brown, S.P. Brown, L. Emsley, W.J. de Grip, H.J.M. de Groot, J. Lugtenburg and M.H. Levitt, Measurement of ${ }^{13} \mathrm{C}-{ }^{13} \mathrm{C}$ $J$-couplings in the rhodopsin chromophore by double-quantum solid-state NMR, to be published.

[49] A. Brinkmann, M. Edén, Second order average Hamiltonian theory of symmetry-based pulse schemes in the nuclear magnetic resonance of rotating solids: application to triple-quantum dipolar recoupling, J. Chem. Phys. 120 (2004) 11726-11745. 